\title{
The Inflammatory Aspect of Male and Female Pattern Hair Loss
}

This article was published in the following Dove Press journal:

Journal of Inflammation Research

\author{
Nadia Peyravian (1D) \\ Sapna Deo' \\ Sylvia Daunert ${ }^{\prime}{ }^{\prime}$ \\ Joaquin J Jimenez ${ }^{1,2}$ \\ 'Department of Biochemistry and \\ Molecular Biology, University of Miami \\ Miller School of Medicine, Miami, FL, \\ USA; ${ }^{2}$ Dr. Phillip Frost Department of \\ Dermatology \& Cutaneous Surgery, \\ University of Miami Miller School of \\ Medicine, Miami, FL, USA
}

\begin{abstract}
Male and female pattern hair loss (MPHL and FPHL, respectively), is the most common cause of hair loss affecting nearly 80 million people in the US, yet treatment options remain limited and lacking. As the need for more effective therapeutics remains unmet, this perspective offers a unique angle by directing attention to the inflammatory aspect of MPHL and FPHL. Evidence and implications of inflammation as a characteristic feature of MPHL and FPHL are highlighted through evaluation of clinical and quantitative data. Comparable results suggest the presence of significant perifollicular inflammatory infiltrates, such as lymphocytes and histiocytes, as well as the involvement of inflammatory genes, such as CASP7 and TNF, in the presentation of MPHL and FPHL. Resurfacing of the inflammatory aspect in MPHL and FPHL pathogenesis will advance future developments in MPHL and FPHL therapeutic options.
\end{abstract}

Keywords: hair loss, inflammation, male pattern baldness, female pattern baldness

\section{Introduction}

Male and female pattern hair loss (termed MPHL and FPHL, respectively, in this paper), affects approximately 50 million men and 30 million women in the US. ${ }^{1,2}$ There is a $50 \%$ risk of development of MPHL in men over 50, and approximately $40 \%$ of women develop FPHL by the age of $50 .{ }^{3,4}$ As the most common cause of hair loss in men and women, MPHL and FPHL impact the individual's self-esteem, self-image, and quality of life. ${ }^{5}$ The fundamental pathophysiological hallmark of MPHL and FPHL is non-scarring alopecia (hair loss), stemming from a variation in the hair cycle and hair follicle miniaturization, resulting in the subsequent reduction of hair of the scalp. ${ }^{6}$ Traditionally, the hair follicle cycle is divided into three main stages: anagen (active hair growth), catagen (cessation of hair growth), and telogen (follicular rest). ${ }^{7}$ In MPHL and FPHL, however, the anagen phase of this cycle is diminished with every cycle while the telogen phase remains unchanged or even extended, yielding diminished hair growth and follicle miniaturization. ${ }^{8}$ Eventually, the progressive decrease in the anagen phase, and the premature transition of the anagen to catagen phase, leads to hair thinning and inadequate hair growth length for successful projection through the surface of the scalp, leaving an uninhabited follicular pore. ${ }^{1,6,9}$

To date, treatment options are restricted to topical minoxidil and oral finasteride, as the only treatments recognized by the Food and Drug Administration (FDA) for MPHL and FPHL. ${ }^{10}$ Other therapeutic options also include low-level laser therapy and platelet-rich plasma therapy. ${ }^{11,12}$ The effectiveness of these treatments is
Correspondence: Joaquin J Jimenez Molecular Biology, Miller School of Medicine, University of Miami, I0II NW

I5th Street, Miami, FL 33136, USA

Tel +l 3052436586

Email j.jimenez@med.miami.edu
Journal of Inflammation Research 2020:13 879-88| 
limited to partial hair regrowth and the need for more effective treatments remains essential. ${ }^{5,13}$ Resurfacing the inflammatory aspects of MPHL and FPHL will allow for a comprehensive understanding of MPHL and FPHL, as well as pave the way for efficacious and novel therapeutic options.

\section{Inflammation and Hair Loss}

While hair cycle variation and follicular miniaturization are regarded as key features of MPHL and FPHL, it is important to also underline the inflammatory aspect of MPHL and FPHL. For example, chronic inflammation signified by the presence of lymphocytes and histiocytes, was found in approximately half the tissue specimens of the over 300 tissue specimens collected from 23 patients with MPHL. ${ }^{14}$ Significant perivascular infiltration of mast cells was observed in about $40 \%$ of the tissue specimens, suggesting a relationship between inflammation and MPHL. ${ }^{14}$ In a similar study, $36 \%$ of biopsy specimens taken from 106 MPHL patients exhibited moderate to severe inflammation with predominantly lymphocyte and histiocyte inflammatory infiltrates. ${ }^{15}$ In a related study, biopsy specimens obtained from over 400 patients with MPHL and FPHL were used to evaluate the presence of perifollicular inflammation with results indicating that approximately $71 \%$ of the MPHL and FPHL specimen samples exhibited inflammation. ${ }^{16}$

Inflammatory infiltrates have been characterized and suggested in the pathogenesis of MPHL and FPHL as observed by an increase in lymphocytes. ${ }^{17}$ Immunohistochemical analysis of biopsies obtained from 3 males and 1 female with progressive alopecia revealed follicular infiltration of $\mathrm{CD}^{+}{ }^{+} \mathrm{T}$-cells at the bulge of the hair follicle while control specimen showed a sparse number of T cells. ${ }^{17}$ This study concluded that infiltration of inflammatory T-cells at the follicular bulge may be the culprit of the impaired hair cycle and successive hair loss in MPHL and FPHL. Similar evaluation of the role of the inflammatory process in the onset of MPHL was conducted using biopsy specimens from 19 MPHL patients and compared to 6 control subjects. ${ }^{18}$ Histopathology of the specimens revealed significant lymphocytic infiltrates in the MPHL patients in comparison to the control subjects. More recently, the role of inflammation in FPHL was studied using scalp biopsies from 52 women with female pattern hair loss. ${ }^{19}$ Lymphocytic folliculitis was observed in the obtained biopsies, indicating the presence of inflammation and suggesting lymphocytic infiltrations as a histological feature of MPHL and FPHL. ${ }^{18,19}$

In addition to clinical and histological evidence, the role of inflammation in MPHL pathology has been highlighted using Next-Generation Sequencing. ${ }^{20}$ RNA was isolated from tissue samples of 5 MPHL patients that were classified according to the phenotypic severity of their MPHL using the Hamilton-Norwood scale. The samples were evaluated against a panel of 30 inflammatory and apoptosis genes using targeted RNA-Seq, revealing differential expression of $W N T 7 A, C A S P 7$, and $T N F$ genes. ${ }^{20}$ The overexpression of $W N T 7 A$, a hair growth gene, was observed in the moderate phenotypes of MPHL, while lower expression was exhibited in the severe late-stage phenotype of MPHL, suggesting hair growth cycle suppression in late stages of MPHL. ${ }^{20}$ Both CASP7 and $T N F$, genes central to cellular apoptosis and inflammation, were overexpressed in the moderate degrees of MPHL, indicating involvement of these genes in the early stages of MPHL. ${ }^{20}$

Therapeutic management of the inflammatory aspect of MPHL has also been shown to be beneficial. ${ }^{21}$ In this study, 20 MPHL subjects with hair loss and pruritus, an inflammatory itching of the scalp, were given lotions consisting of antimicrobial and antifungal agents including triclosan and piroctone olamine, to use regularly for a period of 18 months. ${ }^{21,22}$ The pruritus cleared up during the course of the treatment. ${ }^{21}$ Biopsies taken every 6 months during the course of the study revealed a decrease in activated $T$ cells, suggesting targeting inflammation as a potential approach for treating MPHL. ${ }^{21}$

\section{Conclusion}

The inflammatory role in the pathogenesis of MPHL and FPHL is supported by clinical, histological, and sequencing findings. Perifollicular infiltration of inflammatory cells such as lymphocytes, histocytes, and mast cells is exhibited in biopsy samples of MPHL and FPHL patients. Significant follicular infiltration of $\mathrm{CD}^{+}$T-cells has also been observed as a source for impairment of the hair cycle and the consequential hair loss in MPHL and FPHL. RNA sequencing data signifies differential expression of inflammatory genes in MPHL pathogenesis. Conclusively, these findings suggest inflammation is elemental to MPHL and FPHL pathology and reinforce the need to address inflammation in prospective therapeutic developments. As the inflammatory aspect presents a promising therapeutic approach for slowing down hair loss, targeting 
inflammation may be used to further the efficacy of existing treatments. ${ }^{21,23}$ Future directions should include targeting inflammation for enhancing MPHL and FPHL management.

\section{Acknowledgments}

This work was supported by the National Institutes of Health (R01 HL149452, R01GM114321, R01GM127706, R01MH104656, R01MH110415, HL126559, DA039576, DA040537, and DA044579) and the National Science Foundation (CHE-1506740, CBET-1841419). SD thanks the Miller School of Medicine of the University of Miami for the Lucille P. Markey Chair in Biochemistry and Molecular Biology.

\section{Disclosure}

The authors report no conflicts of interest in this work.

\section{References}

1. Ramos PM, Miot HA. Female pattern hair loss: a clinical and pathophysiological review. An Bras Dermatol. 2015;90(4):529-543. doi:10.1590/abd1806-4841.20153370

2. NIH. Androgenetic Alopecia. In: Medicine UNLo, editor. Genetics Home Reference. USA.gov; 2020.

3. Famenini S, Slaught C, Duan L, Goh C. Demographics of women with female pattern hair loss and the effectiveness of spironolactone therapy. J Am Acad Dermatol. 2015;73(4):705-706. doi:10.1016/j. jaad.2015.06.063

4. Afifi L, Maranda EL, Zarei M, et al. Low-level laser therapy as a treatment for androgenetic alopecia. Lasers Surg Med. 2017;49 (1):27-39. doi:10.1002/lsm.22512

5. York K, Meah N, Bhoyrul B, Sinclair R. A review of the treatment of male pattern hair loss. Expert Opin Pharmacother. 2020;21 (5):603-612. doi:10.1080/14656566.2020.1721463

6. Cranwell W, Sinclair R. Male androgenetic alopecia. In: Feingold KR, Anawalt B, Boyce A, et al., editors. Endotext. South Dartmouth (MA); 2000.

7. Baker RE, Murray PJ. Understanding hair follicle cycling: a systems approach. Curr Opin Genet Dev. 2012;22(6):607-612. doi:10.1016/j. gde.2012.11.007

8. Zhou L, Wang H, Jing J, Yu L, Wu X, Lu Z. Morroniside regulates hair growth and cycle transition via activation of the Wnt/beta-catenin signaling pathway. Sci Rep. 2018;8(1):13785. doi:10.1038/s41598018-32138-2

Journal of Inflammation Research

\section{Publish your work in this journal}

The Journal of Inflammation Research is an international, peerreviewed open-access journal that welcomes laboratory and clinical findings on the molecular basis, cell biology and pharmacology of inflammation including original research, reviews, symposium reports, hypothesis formation and commentaries on: acute/chronic inflammation; mediators of inflammation; cellular processes; molecular
9. Van Scott EJ, Ekel TM. Geometric relationships between the matrix of the hair bulb and its dermal papilla in normal and alopecic scalp. J Invest Dermatol. 1958;31(5):281-287. doi:10.1038/jid.1958.121

10. Adil A, Godwin M. The effectiveness of treatments for androgenetic alopecia: a systematic review and meta-analysis. $J$ Am Acad Dermatol. 2017;77(1):136-41 e5. doi:10.1016/j.jaad.2017.02.054

11. Darwin E, Heyes A, Hirt PA, Wikramanayake TC, Jimenez JJ. Lowlevel laser therapy for the treatment of androgenic alopecia: a review. Lasers Med Sci. 2018;33(2):425-434. doi:10.1007/s10103017-2385-5

12. Cervantes J, Perper M, Wong LL, et al. Effectiveness of platelet-rich plasma for androgenetic alopecia: a review of the literature. Skin Appendage Disord. 2018;4(1):1-11. doi:10.1159/000477671

13. Suchonwanit $P$, Thammarucha S, Leerunyakul K. Minoxidil and its use in hair disorders: a review. Drug Des Devel Ther. 2019; 13:2777-2786. doi:10.2147/DDDT.S214907

14. Lattanand A, Johnson WC. Male pattern alopecia a histopathologic and histochemical study. J Cutan Pathol. 1975;2(2):58-70. doi:10. 1111/j.1600-0560.1975.tb00209.x

15. Whiting DA. Diagnostic and predictive value of horizontal sections of scalp biopsy specimens in male pattern androgenetic alopecia. J Am Acad Dermatol. 1993;28(5 Pt 1):755-763. doi:10.1016/01909622(93)70106-4

16. Whiting DA. Chronic telogen effluvium: increased scalp hair shedding in middle-aged women. $J$ Am Acad Dermatol. 1996;35 (6):899-906. doi:10.1016/s0190-9622(96)90113-9

17. Jaworsky C, Kligman AM, Murphy GF. Characterization of inflammatory infiltrates in male pattern alopecia: implications for pathogenesis. Br J Dermatol. 1992;127(3):239-246. doi:10.1111/ j.1365-2133.1992.tb00121.x

18. Sueki H, Stoudemayer T, Kligman AM, Murphy GF. Quantitative and ultrastructural analysis of inflammatory infiltrates in male pattern alopecia. Acta Derm Venereol. 1999;79(5):347-350. doi:10.1080/ 000155599750010238

19. Magro CM, Rossi A, Poe J, Manhas-Bhutani S, Sadick N. The role of inflammation and immunity in the pathogenesis of androgenetic alopecia. J Drugs Dermatol. 2011;10(12):1404-1411.

20. Martinez-Jacobo L, Ancer-Arellano CI, Ortiz-Lopez R, et al. Evaluation of the expression of genes associated with inflammation and apoptosis in androgenetic alopecia by targeted RNA-seq. Skin Appendage Disord. 2018;4(4):268-273. doi:10.1159/000484530

21. Ge Piérard CP-F, Nikkels-Tassoudji N. Improvement in the inflammatory aspect of androgenetic alopecia. A pilot study with an antimicrobial lotion. J Dermatolog Treat. 1996;7(3):153-157. doi:10.31 09/09546639609086877

22. Zeidler C, Pereira MP, Huet F, Misery L, Steinbrink K, Stander S. Pruritus in autoimmune and inflammatory dermatoses. Front Immunol. 2019;10:1303. doi:10.3389/fimmu.2019.01303

23. Kligman AM. The comparative histopathology of male-pattern baldness and senescent baldness. Clin Dermatol. 1988;6(4):108-118. doi:10.1016/0738-081x(88)90074-0 mechanisms; pharmacology and novel anti-inflammatory drugs; clinical conditions involving inflammation. The manuscript management system is completely online and includes a very quick and fair peerreview system. Visit http://www.dovepress.com/testimonials.php to read real quotes from published authors. 